\title{
SKYLIGHT LIGHT INTENSITY DATA LOGGING SYSTEM
}

\author{
A. UDINA and LC JAIN* \\ ICES, University of South Australia Warrendi Road, The Levels \\ South Autralia, 5095 Australia
}

(Received 12 February 1996; In final form 29 March 1996)

\begin{abstract}
The design of a skylight intensity data logging system is presented. The proposed system is able to accept 32 inputs in two 16 input blocks with capabilities for further expansion. A typical application would be to simultaneously monitor real time change in light intensity in two skylight units, over a chosen periods of minutes, hours or days.
\end{abstract}

\section{INTRODUCTION}

Skylights and skylight systems have grown in size and flexibility over the last 15 years. Many so-called improved systems have gained market attention and the quest is always out to improve the useful light capturing abilities of skylights. Yet there has been very little if any generally published scientific data on the light capturing and transmitting capabilities of the various systems on the market. Qualitatively one system is pronounced as being better than another system, but it is never stated by how much.

As a first step in addressing this gap in the industry knowledge, a light intensity data logging system was designed for the surveillance of light intensity within a skylight duct. Although data logging systems are not new and indeed lux meters have been around for a long time, the problem posed in this situation was the large range of light intensities encountered. The intensity ranges from a few lux to approximately 100,000 lux or 5 decades.

\footnotetext{
${ }^{*}$ Corresponding author.
} 
In general, lux meters that are capable of measuring over that range have two or three ranging resistors that provide the correct ranging. These resistors are generally positioned in a feedback circuit of a current-tovoltage converter. The problem with using this system is that every ranging change will produce a discontinuity across which the output will also be discontinuous. This is due to the different resistors not possessing the same characteristics; hence when a resistor is changed the circuit characteristics and thus the transfer function is also altered. No matter how good the individual elements are, these effects will always be encountered.

This paper describes a design utilizing known technology in providing a simple solution to the described design limitation. Furthermore, this design is able to accept 32 inputs in two 16 input blocks with capabilities for further expansion. A typical application would be to simultaneously monitor real time change in light intensity in two skylight units, over a chosen period of minutes, hours, or even days. The system is portable and can be plugged into the printer port of any IBM compatible Personal Computer. It can also monitor the performance of a permanently installed skylight system.

\section{THE PRINCIPLE OF OPERATION}

Figure 1 shows the system in block diagram form. As may be observed, the system is simple in design with principally four sections: The sensor block, multiplexer block, logarithmic amplifier block, and analog to digital converter block.

The sensor unit comprises the light sensitive diodes, which act as the transducer element. They are reversed biased and can be formed into a bank of diodes. The diode outputs are fed into a 16 to 1 multiplexer. Selection is computer controlled. The output of the multiplexer is used as the input to a logarithmic amplifier. This amplifier has an output of 1 volt per decade.

The output of the logarithmic amplifier is passed into a 10 bit analogto-digital converter (ADC). The input voltage range of the converter is 5 volts, thus giving the logarithmic amplifier a range of 10 to $10^{5}$ lux, or 5 decades. This was deemed adequate for a skylight system. The output of 


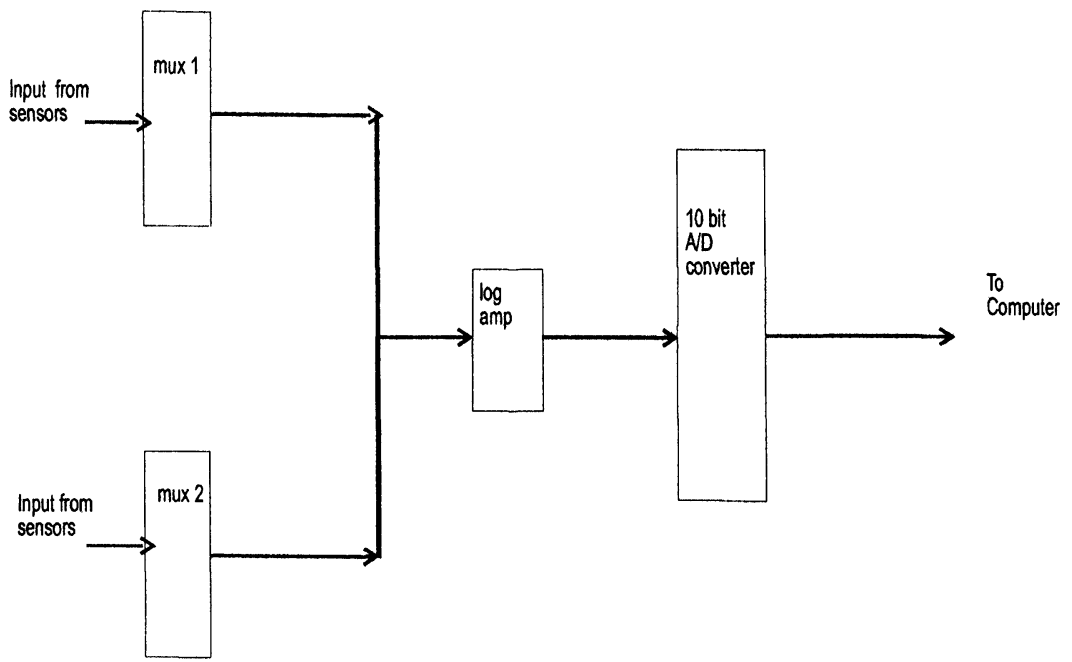

FIGURE 1 Block Diagram of Sensor Circuit.

the ADC is then passed in 4 bit words to a Personal Computer. Here the data is stored for later processing.

\section{DETAILED DESCRIPTION}

\section{Sensor Elements}

The sensor elements used for this application are the BPW21 photo diode. The principal reason why this diode was used is because its spectral range is 350 to $750 \mathrm{~nm}$, which is a very close match to the human eye, in order to measure the visible (to the human eye) light intensity of the skylight. Another important feature is that the short circuit current versus illumination of the BPW21 is very linear over a 10 decade range (from 0.01 to 100,000 lux or $0.475 \mathrm{~mW} / \mathrm{m}^{2}$ to $4.75 \mathrm{~kW} / \mathrm{m}^{2}$ ), making it ideal for our application. The BPW21 also has a good viewing angle of 100 degrees. The temperature stability over the diodes dynamic range is $0-70^{\circ} \mathrm{C}$, which is a suitable range for skylight ducts, since the temperature inside them may exceed $60^{\circ} \mathrm{C}$ in midsummer. Another very good reason for this diode's choice is that it is readily available. 
The connection of the BPW21 was in the standard way, which is reverse biased into a short circuit. In this way, the current was directly proportional to the illumination. The sensitivity of the BPW21 is typically 7 $\mathrm{nA} / \mathrm{lux}$, hence over the illumination range previously described this would give a current range of $70 \mathrm{pA}$ to $0.7 \mathrm{~mA}$. In actuality, the lower bound of the current range is the dark current, which is the current that flows through the diode when it is in total darkness. In the case of the BPW21, the dark current is $2 \mathrm{nA}$, hence the actual workable range of the photo diode would be $2 \mathrm{nA}$ to $0.7 \mathrm{~mA}$. Hence, the cathode was connected to a multiplexer input terminal with the anode going to ground.

Since the skylight ducts into which the sensor array was to be mounted were round, then the sensors were mounted on a nylon grid supported in a round ring. There were sixteen sensors in the array. This ring was affixed at any point inside the skylight shaft by means of four grub screws. In this way, the array could be positioned anywhere in the duct.

To test the suitability of the BPW21, a test circuit as depicted in Figure 2 was implemented. As is shown, the photo diode was dc coupled to the current to voltage converter, with the anode of the photo diode connected to the inverting input of the operational amplifier. This produced a zero bias across the photo diode. The photo diode thus saw the resistor $R_{f}$ as a high resistance load impedance. Since the non-inverting input of the opamp was grounded, then due to the high gain and feedback action of the amplifier the op-amps non-inverting input became a virtual earth. So when light fell on the diode a current was generated; this current flowing through the load resistance $R_{f}$ created a voltage at the output of the current to voltage converter.

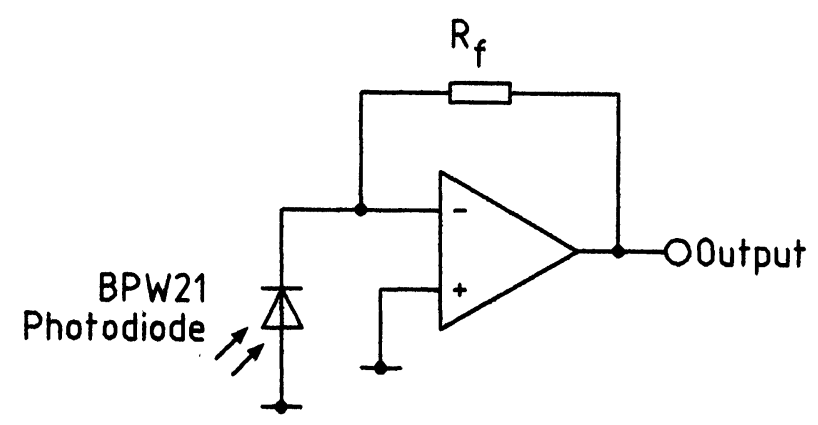

FIGURE 2 Current to Voltage Converter. 


\section{Multiplexers}

There were a number of considerations that were needed for the multiplexers, which were as follows:

1) Number of channels. Since we had 16 sensors to a block, we needed 16 inputs. The question was to use either a 16 channel multiplexer or two 8 channel multiplexers. Price expediency dictated the two 8 channel option.

2) Dynamic Range. As we established earlier, the output from the sensors could range from $2 \mathrm{nA}$ to $0.7 \mathrm{~mA}$, thus we needed analog multiplexers, which would be capable of handling the very low currents.

3) Degree of Crosstalk. Again, since we were dealing with low currents we needed a multiplexer that was quiet enough to allow the input for a channel to not be affected by what may be on any other channel.

4) Degree of Transfer error. As with the previous considerations, the noise figure of the multiplexer would have to be low enough so as to not degenerate the signal beyond recognition.

The multiplexer chosen was the MUX4067. These devices are cheap, popular, and pass all the selection criteria.

One main area of concern was the effect that the input impedance and associated capacitance of the multiplexer switching would have on the small currents employed. The MUX4067 has a channel-on resistance of approximately $330 \mathrm{ohms}$. Since the currents generated were small, it was considered that this resistance would probably have only a negligible effect on the currents. This was found to be the case when tests were carried out on the pre- and post-multiplexer signal amplitudes.

To provide closer correlation between time and channel selected, the switching of the multiplexers was staggered, i.e., channel 1 of multiplexer 1 was sampled first then channel 1 of multiplexer 2 was sampled next, instead of first cycling through the 16 channels of multiplexer 1 and then cycling through the 16 channels of multiplexer 2 . This was effected by first selecting the channel and then toggling the multiplexer control lines.

\section{Logarithmic Amplifier}

This amplifier provided the amplification and conversion from the current source to a voltage source, at a rate of 1 volt per decade of lux input. Since 
the lux range that we were considering was 5 decades, the logarithmic amplifier output had a range of 5 volts. This was so designed so that the interface to the ADC would be straight forward.

Figure 3 provides a circuit diagram of the logarithmic amplifier. As can be seen, it is a rather straight forward amplifier depicted in a range of references (Linear applications handbook). The operational amplifiers used are LM11c. The input was negative, hence the transistors $\operatorname{Tr} 1$ and $\operatorname{Tr} 2$ were 2N4058's. To determine which offset would be the best, the Transfer function of the logarithmic amplifier was coded in Matlab and a model was produced. This was done primarily to observe the zero crossing, as this would cause problems in actual applications. Figure 4 demonstrates the output of the model. It can be seen that the zero crossing for a reference voltage of 10 volts is $10^{-4}$ lux compared with $10^{-5}$ lux for a 1 volt reference voltage. This is of course the type of behavior that one would expect, but because of the logarithmic nature of the circuit, to push the

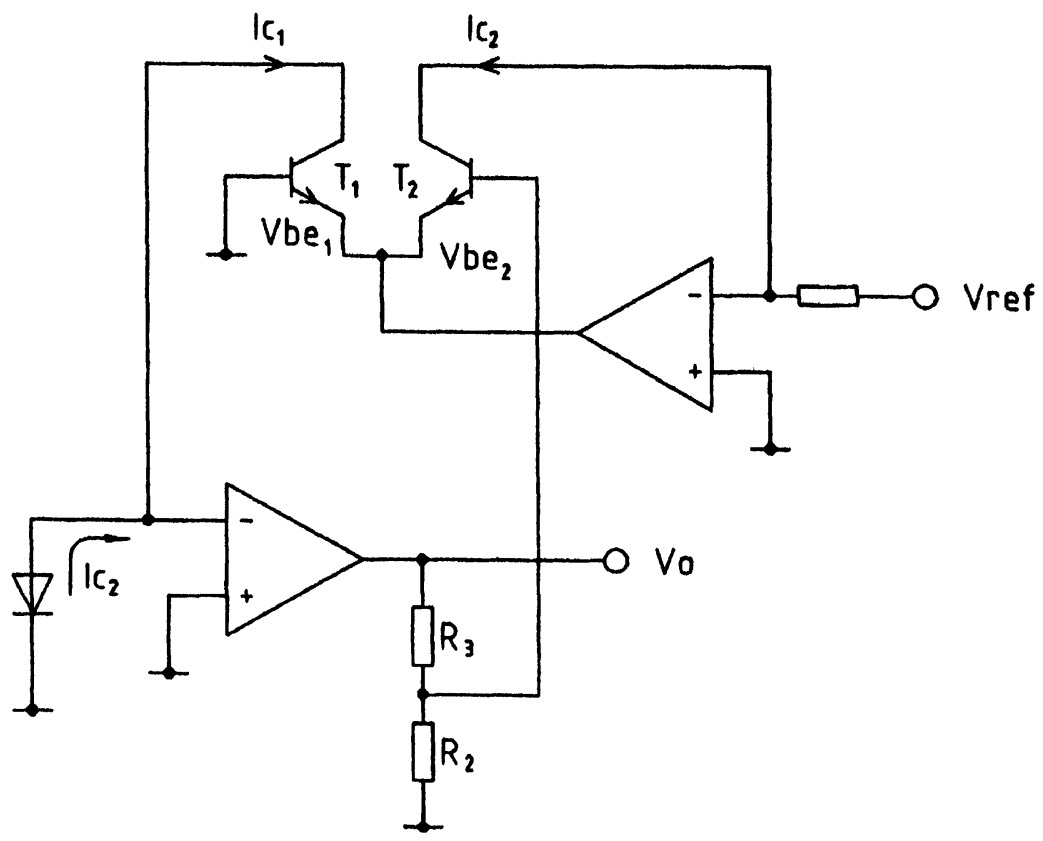

FIGURE 3 Circuit Diagram of Logarithmic Amplifier. 

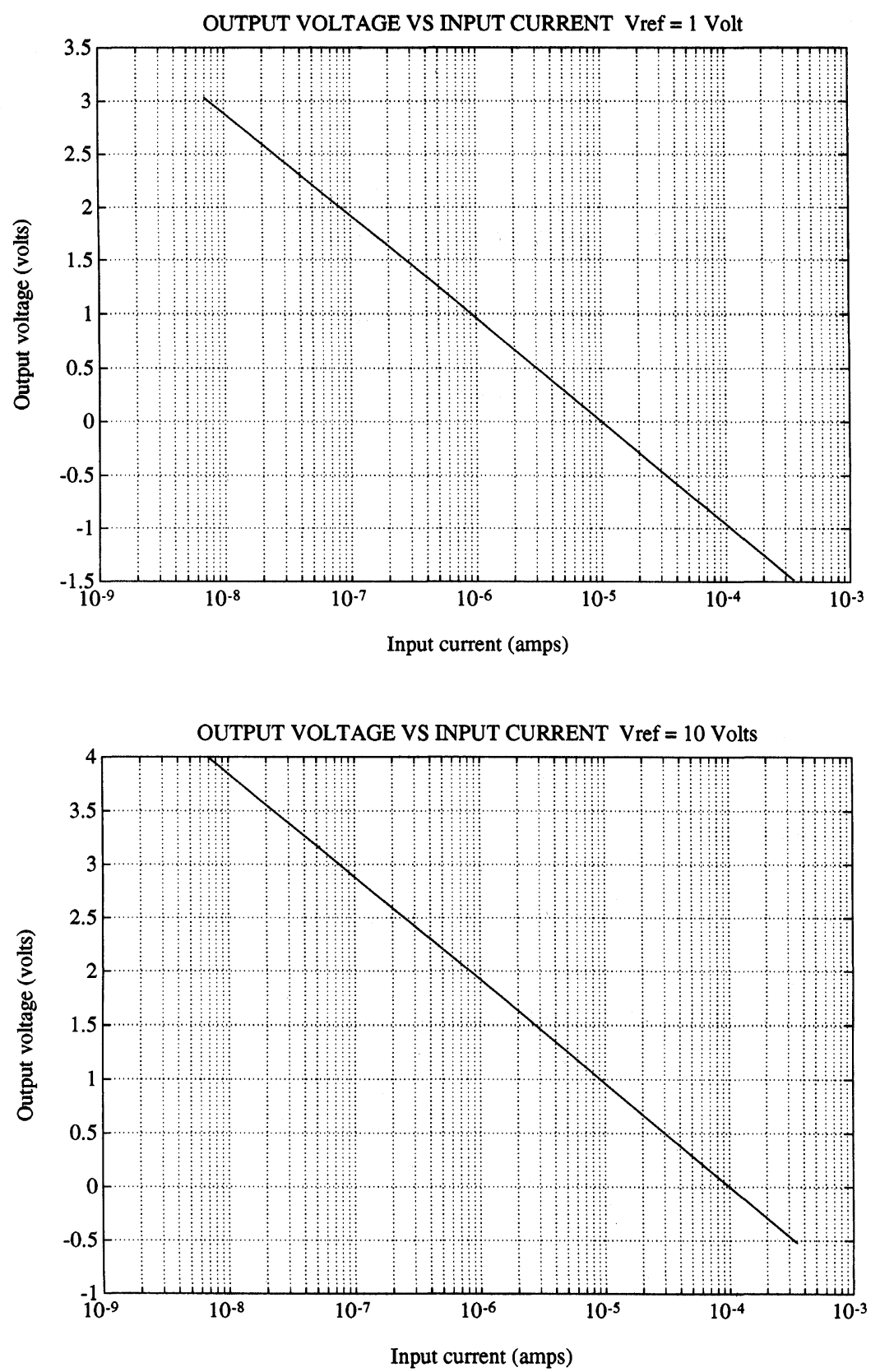

FIGURE 4 
zero crossing to $10^{-3}$ lux would necessitate a reference voltage of 100 volts, which was not possible, hence it was decided to maintain a reference voltage of 10 volts.

\section{DERIVATION OF $V_{0}$ AS A FUNCTION OF IC2}

It is clear from the circuit diagram in Figure 3 that

$$
-V 1+\text { Vbe } 2-V b e 1=0
$$

Now let us find

$$
\frac{I c 1}{I c 2}
$$

Also

$$
I c 1=\frac{V r e f}{R 1}
$$

Thus we obtain

$$
\frac{I c 1}{I c 2}=\frac{V r e f}{I c 2 * R 1}
$$

We have from the Ebers-Moll equation that

$$
I c 1=I s c * \exp \left(\frac{\mathrm{V} b e 1}{\mathrm{~V} t}\right)
$$

and

$$
I c 2=I s c * \exp \left(\frac{V b e 1}{V t}\right) \quad * \text { NOTE } * \quad \text { Take transistors to be the same }
$$

So substitute into (2) to obtain 


$$
\frac{I c 1}{I c 2}=\frac{I s c * \exp \left(\frac{V b e 2}{V t}\right)}{I s c * \exp \left(\frac{V b e 1}{V t}\right)}
$$

This gives

$$
\frac{I c 1}{I c 2}=\exp \left(\frac{V b e 2}{V t}\right) * \exp \left(-\frac{V b e 1}{V t}\right)
$$

So get

$$
\frac{I c 1}{I c 2}=\exp \left[\frac{1}{V t}(V b e 2-V b e 1)\right]
$$

From (1) we have that

$$
\text { Vbe } 2-\text { Vbe } 1=V 1
$$

So substituting this into (4) we get

$$
\frac{I c 1}{I c 2}=\exp \left(\frac{V 1}{V t}\right)
$$

Now let us substitute (5) into (3) to get

$$
\frac{V r e f}{I c 2 * R 1}=\exp \left(\frac{V 1}{V t}\right)
$$

Taking the $\log$ of both sides gives us

$$
\frac{V 1}{V t}=\ln \left(\frac{V r e f}{I c 2 * R 1}\right)
$$

or

$$
\frac{V 1}{V t}=-\ln \left(\frac{I c 2 * R 1}{V r e f}\right)
$$


Now from the circuit diagram we can see that V1 and V0 are related by the voltage divider in that

$$
V 1=\frac{R 2}{R 2+R 3} * V 0
$$

Using equations (6) and (7) we have

$$
\frac{V 0}{V t} * \frac{R 2}{R 2+R 3}=-\ln \left(\frac{I c 2 * R 1}{V r e f}\right)
$$

Therefore we have

$$
V 0=-V t * \frac{R 2+R 3}{R 2} * \ln \left(\frac{I c 2 * R 1}{V r e f}\right)
$$

We know that Vt, R1, R2, R3, and Vref are constants. We can therefore see that $\mathrm{V} 0$ is a function of the negative logarithm of Ic2.

\section{ANALOG TO DIGITAL CONVERTER}

Because of the slowly changing nature of the input, the sample and hold devices that were normally associated with analog to digital converters were not needed. Similarly this meant that speed was not a major concern with the ADC. The successive Approximation ADC was found to be a good overall candidate. It was fast, has good resolution and importantly the internal logic is cleared after each conversion. This meant that there was no conversion dependence between successive conversions. This type of converter was also very good at converting filtered low noise inputs. The converter that was finally decided on was the ADC1025.

To function correctly, the ADC needed to be clocked. Since there were no clock lines incorporated in the design, the clocking had to come via the internal ADC clock. The device data handbook suggested that the ADC be clocked at a frequency of $600 \mathrm{kHz}$. The clock used an external RC circuit, so the chosen frequency was arrived at with a resistor value of $10 \mathrm{kohms}$ and a capacitance value of $150 \mathrm{pF}$. 
The ADC also needed a reference voltage to define the voltage span over which it would quantize its input. In our case, the voltage span was 5 volts. This was achieved by tying the reference line to the 5 volt supply rail. This obviated the need to have the supply rails regulated so that amplitude changes would be kept to a minimum. Testing demonstrated that the supply rails were adequate as a reference line.

\section{FEEDING DATA TO THE COMPUTER}

The interface to the computer was through the LPT 1 or printer port. Now, because of the configuration of the printer port, it could only accept a 4 bit parallel stream. Hence, this meant that the data needed to be broken up into two 4 bit words and one 2 bit word. This was achieved with the use of three LS125 buffers. The ten ADC outputs were divided into two sets of 4 bit words, each feeding into separate buffers and one 2 bit word feeding into the third buffer. The microprocessor then cycled through the buffers reading the 10 bits in the following sequential manner. It first enabled the high nibble, then the middle nibble, and finally the last two significant bits. In this way the 10 bits were sent through 4 lines, which were then reconstructed in the computer memory.

\section{CONCLUSION}

This paper presents the design of a data logger system to measure light intensity in the duct of skylights. The design is simple, inexpensive, and has the capability of measuring intensity ranging from 10 lux to $10^{5}$ lux without having to resort to range changing resistors.

\section{Acknowledgment}

We would like to thank Kim Kemeny and KimAhn Loung for their work in the production and testing of the components and devices. 

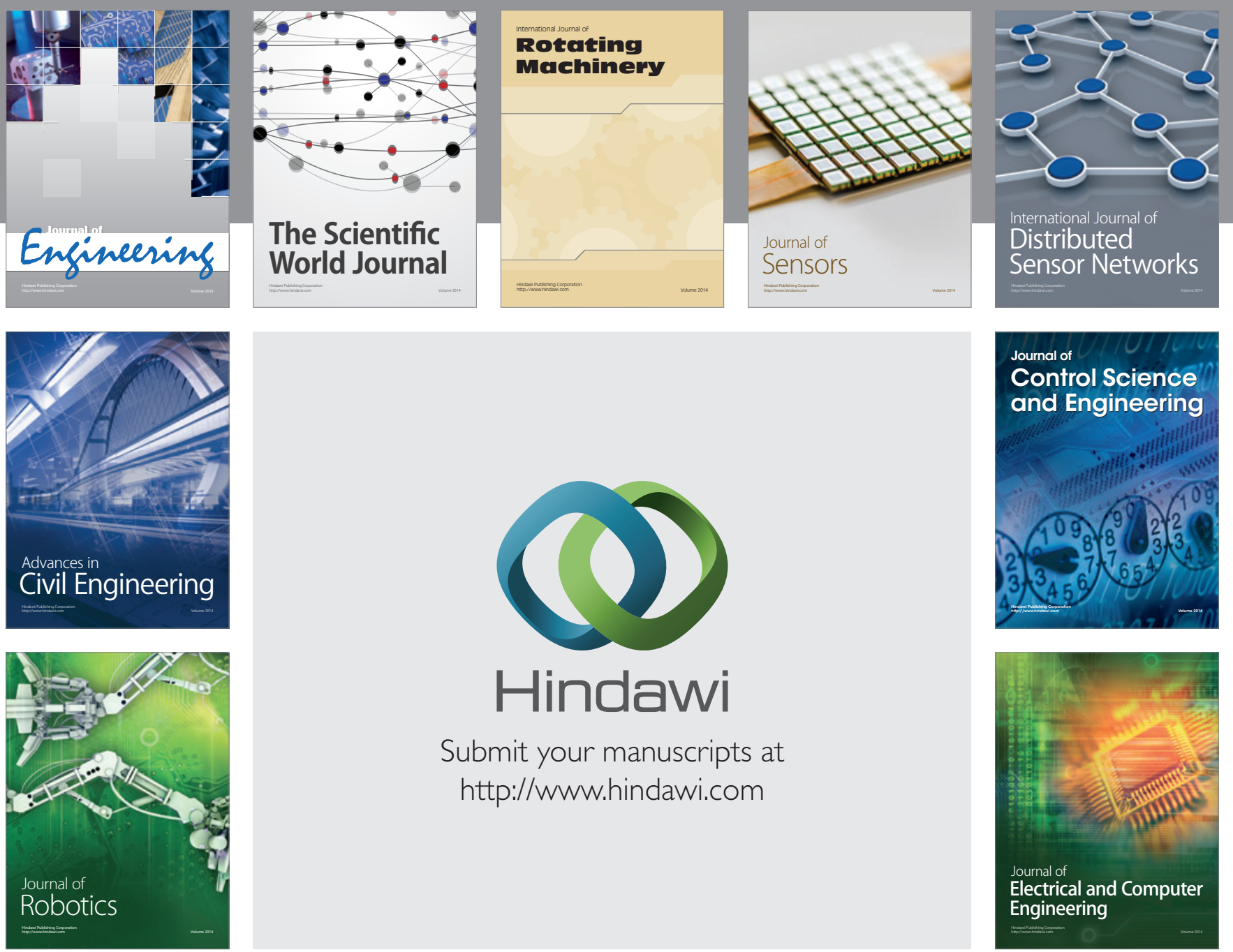

Submit your manuscripts at

http://www.hindawi.com
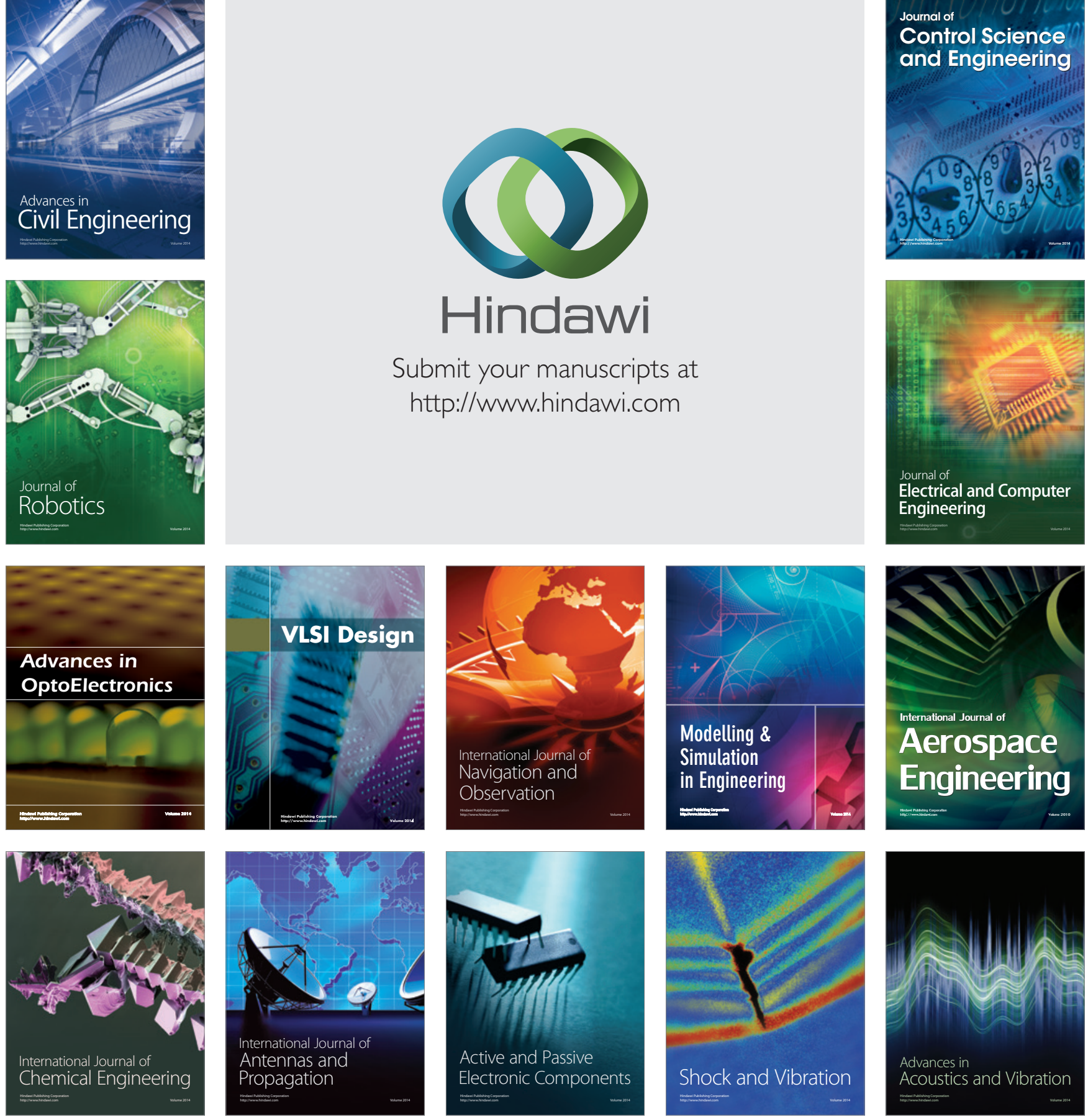